\title{
Probiotics bacteria, properties, and their applications
}

\author{
Rania Mohammed Sabri Sultan \\ Department of Biological Sciences, Faculty of Science, King Abdulaziz, University Jeddah, 21589, Saudi Arabia
}

Correspondence Author: Rania Mohammed Sabir Sultan, Department of Biological Sciences, Faculty of Science, King Abdulaziz, University Jeddah, 21589, Saudi Arabia

E-mail:- dr_sultania@yahoo.com

Received date: 16 August. 2019, Accepted date: 16 Nov. 2019, Online date: 1December 2019

Copyright: 2019 C Rania Mohammed Sabir Sultan., This is an open-access article distributed under the terms of the Creative Commons Attribution License, which permits unrestricted use, distribution, and reproduction in any medium, provided the original author and source are credited.

\begin{abstract}
Probiotics have been a useful component of the microbiota for centuries because of its health benefits to the host. However, the contribution of probiotics to the modulation of immunological, respiratory and gastrointestinal functions has recently been recognized and appreciated by scientists. Probiotics, such as Escherichia coli and lactic acid bacteria, are currently used or studied to prevent or treat various intestinal diseases, including inflammatory bowel disease, constipation, and colon cancer. Building natural probiotics to produce immunomodulatory molecules can help further increase the benefits to the host. This article analyzes some of the mechanisms of action of probiotics and achievements in the rational design of probiotics. In industrialized countries, functional foods have become a daily diet and add to the generally recognized nutritional effects of proven and potential health benefits. Currently, the most important and widely used functional food compounds are probiotics and prebiotics, or collectively they are called "symbiotic" and in a healthy manner, Therefore, The aim of this work overview on of probiotics bacteria, properties, and their potential applications for functional foods to improve the health and nutrition of society
\end{abstract}

Keywords: Probiotics, bacteria properties, applications, human.

\section{INTRODUCTION}

Probiotics are defined as living bacteria, the appropriate host sufficiently introduced and beneficial to health [1]. The first observation of the positive characteristics of certain bacteria is attributed to the Nobel Prize winner Eli Melnikov, considered the grandfather of modern probiotics. At the beginning of the 20th century, Melnikov discovered that "healthy bacteria", especially lactic acid bacteria, could have a positive effect on digestion and the immune system [2]. Most of the microorganisms previou sly recognized as probiotics are Gram-positive, with Lactobacillus and Bifidobacterium being the main species used to treat intestinal disorders [3]. However, some Gram-negative substances are also used as probiotics. The best example of this group is E. coli [4], also known as "Mutaflor", known in Germany for many years for the treatment of chronic constipation [5] and colitis. Numerous studies conducted over the last century have shown that probiotic bacteria play an essential role in the modulation of immune, respiratory and gastrointestinal functions [6]. Besides, there are probiotics. It has been shown that its protective role is to compete directly with intestinal pathogens by releasing antibacterial agents such as bacteriocins [7] or metabolites such as acetic acid and lactic acid [8]. Although most studies have advanced on empirical probiotics, a new one could come from research on the interaction between coma microorganisms and table microorganisms (the microbiota) between the pathogen and the host. Understanding colon colonization mechanisms, both in terms of healthy and inflammatory processes, is crucial for the development of probiotics for a specific application. In this article, we will discuss some recent developments related to the mechanisms of action of probiotics and their use for the delivery of molecules to specific host species. In addition, dairy products have been invented to look like good nutritious food. These probiotic dairy products have a positive effect on the host by surviving and enhancing the implantation of live microbial food supplements into the stomach - intestines - flora, selective growth or catabolism stimulation using one or more choices. Bacteria are good for intestinal health and improve the microbial balance of the gastrointestinal tract. The aim of this study to the overview of probiotics bacteria, properties, and potential applications for functional foods to improve the health and nutrition of society [9]. 


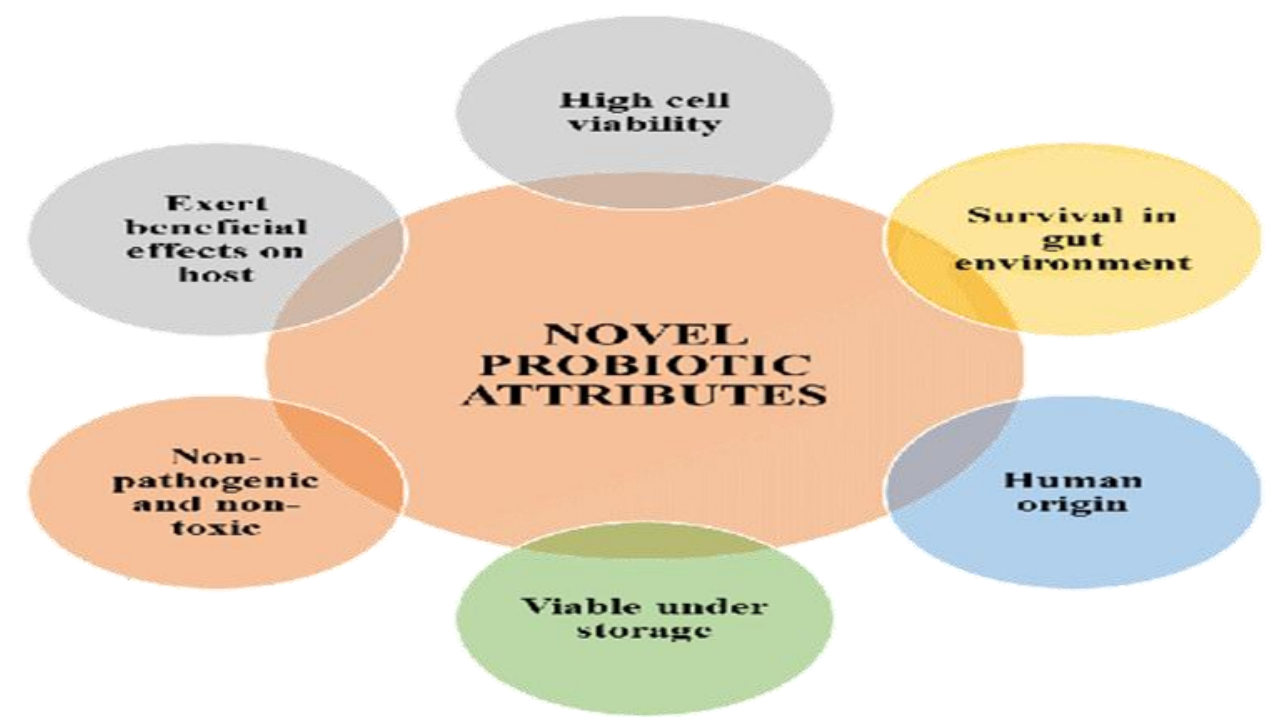

Figure 1: Some of the properties of novel probiotic by [10].

\section{DIGESTIVE PATH MAN AND MICROBIOTA}

The human digestive tract includes several ecological niches of several populated bacterial species that have established a symbiotic relationship with the host. This bacterial population, also known as the intestinal microbiota, plays an important role in the development of the intestinal immune system, the digestion of food, the production of short-chain fatty acids and essential vitamins and the resistance to colonization by pathogenic microorganisms [11]. A human microbial includes many types of bacteria, some of which are not cultured and are therefore poorly known or characterized. In fact, the complexity of the microbiota has only been fully understood in the last decade through deep sequencing, genomics, and metagenomics. The distribution of intestinal microflora varies according to the three main areas of the stomach: the intestines and the gastrointestinal tract: (1) placed stomach, 102 colony-forming units $(\mathrm{CFU}) / \mathrm{ml}$, including lactobacilli and streptococci; (2) distal ileum and distal ileum 102-103 $\mathrm{CFU} / \mathrm{ml}$ of bacteria, including Escherichia coli, Klebsiella, enterococci and Bacteroides; and (3) the colon, which is the largest microbial population in the body, at 1010-1012 CFU/ ml [12]. It should be noted that each organism has an "area of exposure to specific bacteria", influenced by several factors, including the average maternal environment, host genotype, diet and antibiotics [13]. But even if the composition of the microbiota from one person to another is grouped into three different groups, it is called enterotypes. These human enterotypes are enriched with Bacteroides, Prevotella or Ruminococcus and produce energy from fermentable substrates present in the colon in different ways: Bacteroides use carbohydrates; Prevotella, the mucins; and Ruminococcus, mucin and sugar [14]. Enterotypes have also been associated with long-term diets [15]. Despite this heterogeneity, Firmicutes and Bacteroidetes are the most common intestinal filaments in all vertebrates, representing 0.90\% of the microbiota, followed by actinobacteria and proteobacteria [16]. The members of the microbiota Phylum Bacteroidetes belong to several species, including the fragile bacteroid, according to the recent immunomodulatory potential resulting from the demonstration of a polysaccharide capsule. On the other hand, the Firmicutes border belongs mainly to the species of the class of Clostridia presented, known for its ability to metabolize fibers and butyrate, a short-chain fatty acid with immunomodulatory formation [17, 9]. Bacillus class bacteria, including Lactobacillus acidophilus and Enterococcus faecalis, form the rest of the border with firmicutes.

Probiotics and their health benefits, as well as applications to develop more safe foods: an overview

Probiotics are defined as "living micro-organisms that, when administered in sufficient amounts, have beneficial effects on health" [18]. Alternatively, probiotics have been defined as live microbial supplements that positively affect the host animal, thereby improving its microbial balance in the intestine [19]. Probiotics were originally used to ensure animal and human health through better modulation of intestinal microflora. Currently, several strains of Lactobacilli and Bifidobacteria are characterized by their usefulness for human consumption to reduce the risk of gastric or intestinal infections or the treatment of such infections [20]. Beneficial effects of using probiotics include improving intestinal health by regulating the microbiota and stimulating and developing the immune system, synthesizing and improving the bioavailability of nutrients, reducing the symptoms of lactose intolerance and reducing the risk of other diseases [21]. The main clinical interest in the use of probiotics has been the prevention and treatment of infections and gastrointestinal disorders [22]. By moving away from the intestinal microflora, it is associated with an increased risk of developing certain diseases; Therefore, modulation of unbalanced root microflora is the cause of probiotic treatment [23]. In addition, the development of adjuvant or replacement therapy based on the bacterium will be of great importance because of the development of pathogenic strains resistant to antibiotics and adverse effects of antibiotics on the flora. protection increases the risk of infection [24]. However, the use of probiotics needs to be further investigated to determine the benefits and possible side effects. While knowledge of the gut microbiota, diet, immunity, and genetics of health and disease has developed in recent years, it can certainly help develop new strains of probiotics with functions. It can also help you understand when to use probiotics and how they affect certain painful conditions. However, it is important that probiotic strains for human use be tested in animals and then in human clinical trials to demonstrate the relevance, safety, and usefulness of probiotics for consumption. Development of human products and functional products. Essential properties for the effectiveness and success of probiotics. It is imperative that the probiotic strain survives where it should be active. For maximum activity, the strain must be 
able to multiply and colonize at this location. In addition, it must also be a tolerant immune system. It must not be pathogenic, allergic or mutagenic / carcinogenic [25]. Probiotics for a person who already has the status "generally considered safe", induce a low risk or are related to the etiology of the disease. The probiotic microorganisms must preferably be of human origin [26], in order to survive and develop under the desired conditions of administration in vivo, and thus to have a low $\mathrm{pH}$ and to be able to withstand concentrations. High bile conjugated and deconjugated. Acid For successful implementation in foods, the probiotic that will be used technologically is a compatible production process. In addition, products containing probiotic bacteria must maintain the sensory properties characteristic of traditional foods. Properties and potential benefits of probiotics. At present, it has been discovered that native microbial communities are highly complex, host-specific, site-specific and that HA has five positive host properties. But the type of micro-organization that plays an important role in these beneficial properties is unclear. Some of the main benefits of probiotics and the health of the supposed mechanisms. There were more probiotic bacteria on the market and the product line containing probiotic bacteria is increasing. Some of the key health characteristics of probiotics are discussed in the following sections. Antimicrobial properties The intestinal microbial community is a complex ecosystem and it is difficult for new organisms to introduce a highly competitive environment. Therefore, organisms capable of producing a growth-inhibiting product from existing organisms have a distinct advantage. The ability of probiotics to establish themselves in the gastrointestinal tract is enhanced by their ability to eliminate their competitors. Some antimicrobial agents containing producer organisms. In various studies in humans and animals, useful microorganisms must be used to improve resistance to colonization on the body surface, such as the gastrointestinal genitourinary tract and the respiratory form bifidobacteria produce acid, acetic acid, and lactic acid in a molar ratio of 3: 2 [27]. Lactobacillus acidophilus and Lactobacillus Casey are products derived from lactic acid, the final products of fermentation. In addition to lactic and acetic acids, probiotic organisms produce other acids, such as acid, hippuric acid, and citric acid. Lactic acid bacteria also produce peroxide from hydrogen, diacetyl, and bacteriocin as antimicrobial agents. These inhibitors create an antagonistic environment of byproducts of foodborne pathogens and parasites. Yoghurt bacteria are said to produce a bacteriocin against probiotic bacteria and vice versa [28].

The anticancer properties of [29] indicate that the administration of L. acidophilus in the diet induces the incidence of chemically reduced colon tumours in the rat. Later, surpassed by the same authors, diet and antibiotics can also produce carcinogens in the colon and tumor-induced chemicals may decrease [30]. These effects appear to be mediated by intestinal microbial communities. A possible mechanism for this rel. Anticancer effects and those associated with intestinal bacterial enzymes inhibit procarcinogen for the conversion of more proximal carcinogens [31, 32]. This approach could be extended in the future by the ability of probiotics to evaluate the growth of organisms of normal origin (intracellular adhesion molecules and integrins), cell adhesion (cadherins), transcription and signal transduction. For Inhibition Some researchers have also found that probiotics reduce the concentration of fecal enzymes (glucosidases, beta-glucuronidase, azoreductase, and nitroreductase) and reduce secondary bile salts and mutagenic agents that are harmful to absorption and may contribute to carcinogenesis. The normal intestinal flora of the colon can influence carcinogenesis by producing enzymes (glucosidases, B-glucuronidase, azoreductase, and nitroreductase) that convert active, direct or carcinogenic carcinogens [33]. The addition of Lactobacillus acidophilus and $L$. casei to the human body decreases the amount of these enzymes [34]. In mice, these bacterial enzymes were inhibited by the administration of Lactobacillus GG [35]. They proposed various mechanisms for both milk bacteria and cancer-inhibiting bacteria, including improving the immune response of the host, altering the metabolism of microbial activity of the intestine within the body. the community. Physics with chemical conditions in the large intestine [36, 31, 32] LAB oral administration has been shown to effectively reduce DNA damage caused by carcinogens in the gastric mucosa and rat colon [37, 38].

\section{Graphical abstract [39].}

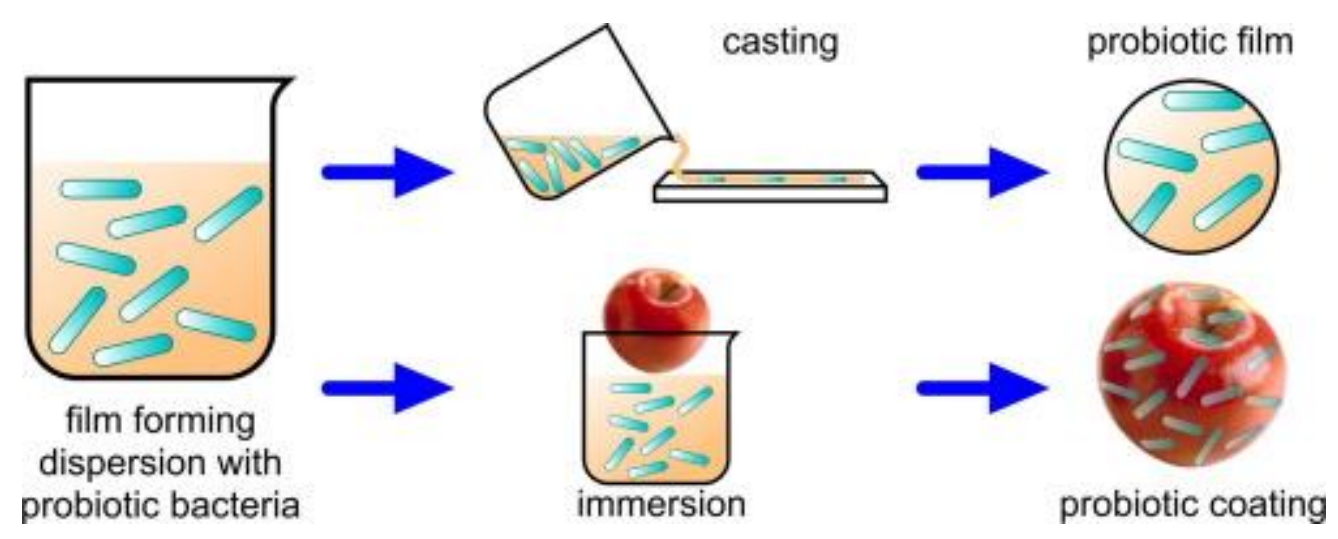

\section{Bifidobacterium}

The microbial composition of the gastrointestinal and intestinal tract (GIT) in the human body changes throughout life. They can describe three distinct stages in almost all people and correspond to each birth when the microbiota begins to colonize the sterile gut, the time of weaning when another diet determines the profound changes that lead to a stable community, which will be present. at elderly people, when they appear new important changes in the microbiota. This review focuses on the microbiota of children from birth to weaning, with a focus on bifidobacterial. The latest Bifidobacterial application documents for infants and young children for the prevention and treatment of intestinal and non-intestinal pathology are described. Potential applications of these probiotic bacteria for the treatment of diseases for which the probiotic approach appears promising based on ongoing in vitro studies are also proposed. Thus, this document provides a comprehensive overview of the role of Bifidobacterium spp. Native and 
managed. In neonates and infants, according to the description of the results, the authors collected reputed scientists obtained in the authors' laboratory. The use of probiotic bacteria, such as bifidobacteria, for the prevention and treatment of disease, is a new area of applied microbiology called "therapeutic microbiology".

The composition of the microbiota in the newborn and factors affecting microbial colonization. The intestines of a newborn resemble the intestines of an animal without germs. Microbial colonization begins shortly after birth and some bacteria appear in the stool [40]. First, the microorganisms are acquired by contact with the mother; Then, the infant is exposed to the effects of the microorganism environment. summarizes the main factors that affect microbial colonization in the intestine. The first bacteria found in most healthy children are facultative anaerobes because the intestinal potential of newborns is positively oxidized/decreased at birth. These bacteria, including Staphylococcus spp., Enterobacteriaceae and Streptococcus spp., Remain predominant in the early days of life. They are mainly isolated after birth in the stool of the newborn. Optional anaerobic bacteria are followed by heavy anaerobic bacteria, such as representatives of the genera Bifidobacterium, Bacteroides, and Clostridium. The hospital room is very important for cesarean colonization of the intestines born to infants. These newborns do not meet the fecal and vaginal microorganisms of the mother and are separated from the mother shortly after birth [41]. In the largest neonatal epidemiological study investigating factors affecting intestinal composition Koala study, [42], anaerobic colonization, particularly Bacteroides spp. In newborns, cesarean section is delayed. But Bifidobacterium spp. Recovery and the presence of Escherichia coli were similar in neonates after cesarean section and cesarean section. In addition, he reports a higher incidence of Clostridium perfringens and Clostridium difficult under cesarean section associated with a cesarean section of neonates. Another important factor affecting the composition of the gut microbiota in newborns is the type of nutrition identified in the koala study. Under the term "breastfeeding", Bacteroides spp. and Bifidobacterium appears within 4 days of birth and 1 week, dominated by fecal microbiota, with $80-90 \%$ of the total number of microbes. In contrast, fecal microbiota in infants fed formula is Bifidobacterium spp., Enterobacteria and Streptococcus spp. The complex. a noticeable difference is that babies fed on food have a lot more Clostridium spp. there is. It's like breastfeeding. An important difference is the relative buffer capacity of the two streams. Breast milk has low buffering capacity compared to formula, resulting in significant differences in colonic pH in breastfed infants, considering formulas 5.1 and 6.5, respectively. Low $\mathrm{pH}$ promotes the growth of bifidobacterial and lactobacilli but inhibits many other bacteria [43]. In addition, recently, several peptides from isolated milk, the growth of more bifidobacterial stimulate the bank. In addition, breast milk contains glycoproteins, glycolipids, fucose, neuraminic acid, lactose, N-acetylglucosamine, and various oligosaccharides, known to have bifidogenic effects [44]. Neonates and adults are usually exposed to microorganisms through food, but with different effects: it is more likely that micro-organisms entering the newborn are more likely to colonize than those reaching the neonate. 'adulthood. It's great to have a stable microbiota. Breast milk is a potential source of microorganisms, although the results so far have been the isolation and identification of potential probiotic bacteria from guests or that the milk of healthy women is not yet definitive. Although the authors are aware that breast milk is difficult to collect and that microbial contamination can never be eliminated, some studies have shown the presence of bifidobacterial that constitutes the life of breast milk [45], [46]. Unlike the newborn, there is little information on the composition of the microbiota in premature infants with respect to individual variability between more than the new - in time and the origin of a number of parameters, such as that the antibiotic, and to study the contradictions increase, but often require a diet of parental support and respiratory diseases, prone to infections and often require antibiotics - treatment, in addition, the gastric $\mathrm{pH}$ of premature babies is superior to the neonatal in time full, probably, from - for - part of the diet and the risk of bacterial infections increases. altering the composition of the intestinal flora of prematurity can with increased risk for ESR patients, serious intestinal gastrointestinal diseases such as necrotizing enterocolitis (NEC) is connected [47, 48].

\section{Applications of Microbial Interactions for Human Welfare}

The combined use of Monday food microbes, such as fungi, filamentous yeasts and LAB, plays a key role in the production of many fermented foods [49]. This co-culture greatly contributes to the organoleptic properties of foods and improves their quality in different ways. For example, it changes the aroma, taste and texture of foods and sometimes increases the shelf life of fermented products [50]. It is also used in combination cultures for the fermentation of bacteria and fungi in various fields of application, particularly in the production of alcoholic beverages, such as wine [51] and beer [52], dairy products. [53] such as cheese and kefir [54] and yeast [55]. There is; however, there is very little research on the interaction of bacteria and fungi at the cellular level in the fermentation process. Yeast cannot be used to improve the use of bacteria and crops because their mechanism of cell interaction has not yet been elucidated [56]. None, however, a more detailed study of LAB's molecular interaction with the production of finished antifungal and fungal metabolites in bakery products, cheese, beer [57]. LAB antifungal compounds products such as organic acids (lactic phenyl, propionic, acetic and lactic acid), hydrogen peroxide, cyclic fatty acid hydroxy dipeptides and reuse, and the protein has not been characterized, and phenolic compounds [58]. In addition, yeast can inhibit the growth of contaminating bacteria from Listeria monocytogenes in spreads [59]. Currently, significant efforts have been devoted to the fermentation process for the development of microorganisms, which have an inhibitory effect on the bacteria and the fungi responsible for the distortions, both in need, without additives. Meals have become more important. In addition, the risk of food spoilage caused by the formation of molds producing mycotoxins or pathogenic bacteria can be minimized by initiating cultures of inhibition. This type of food poisoning represents a reasonable risk to the health of the population and a threat to the economy. Biotransformation and biodegradation of mycotoxins by microorganisms are other recent areas of research used to combat food poisoning [60]. Foods and other foods are often contaminated with mycotoxins, such as aflatoxin, zearalenone, feminizing and trichothecene, which cause toxic reactions when they are subsequently absorbed. These fungal toxins can be easily transformed or even destroyed by various bacteria [61]. It is useful that the microbial degradation process, a thorough critical analysis, is necessary, since not all biotransformation in the detoxification of mycotoxins are necessary. In addition, this examination is necessary to determine the stability of the processed product after human consumption and animal consumption [62]. This may be their utility or perhaps with the definition of time - long term or short term. Although the transformation and potential for bacterial 
degradation previously studied are numerous, it seems to be used in the biotransformation enzymes, which are used by one and the same organism, the greater the potential and if living organisms were found. Treatment [63]. The beneficial effects of microorganisms in a mixed culture (interaction between bacteria and fungi) in food production can also lead to contamination of food and, as a result, significantly affect human health [64]. This text illustrates Tempe Bangko k, a coconut-based dish widely available in Southeast Asia. This product was obtained by fermenting a coconut cake using the fungus Rhizopus anisospores [65, 66].

Perspectives he is important for understanding the response of microorganisms to signals from plant facilities to study these interactions. Whether it is a future in the development of biotechnology with respect to agricultural dependence in genetic modification technology or classical breeding, you need to consider the benefits of plant and plant associations. microbes. The Centers sector is the "traditional" biotechnology of plants around plant breeding and, finally, the choice of properties that have an advantage and measure the microbial ecology of the plant is envisaged by the association. However, the expression of desirable characteristics such as disease resistance, drought, and salt tolerance can also be directly affected by the interaction between certain plant species and their interacting microbial flora partner. Also, possible, the scenario is different, when some kinds may be included interactive connections with microorganisms with undesirable characteristics. A clear interpretation of the genetic basis of interactions between plants and micro-organisms, when examining the mechanism by which a given plant, can selectively determine, allows the "conditioning" of the rhizosphere, properties contributing to more sustainable development. plant, in fact, the natural ground at the base of is suppressor. The expression of a group of biosynthetic genes is often strictly controlled so that fluctuations in environmental conditions occur. In pure cultures, the coding of natural products of biosynthetic genes usually remains silent, so that many metabolic products are often not collected. - the work of a mixed experimental culture, in this case, to eventually activate the use of silence genes provided, so that the potential of metabolites gives rise to a priority for the investigation of power. The fermentation of foods in mixed crops has a significant economic benefit. The cultures are LABs, yeasts, and filamentous fungi. Therefore, their effectiveness is not limited to the mere coexistence of the characteristics of an individual cell with a strain, it is mainly determined by the interaction of the substrate - the level of metabolism of the metabolism. and growth products: factors that enhance or inhibit growth. Advances in the following recent developments in the field of genetic technology have been presented more boldly, both on a model based on inference descriptions. In addition, the study, which was founded, requires an understanding or is, more ecological principles as well for the development of strategies as laboratory strains and artificial ecosystems. Thus, fermented foods provide alternative technologies and resources and are a very appropriate practice. An in-depth study of the current availability of tools for genomic and genetic expansion tools could combine approaches, potential mechanistic and evolutionary solutions, constituting a vital problem. Knowledge of the different types of microbial interactions and their utility provides a fertile platform for the creation of well-organized synthetic microbiomes that can be used in many contexts. Example: the optimal solution for health: problems related to hygiene and heavy metals, etc. In vivo metabolic activity in cells during contact interactions plays essentially a decisive role as "bimotored" of major metabolites, such as volatile organic compounds (VOCs) and essential metabolites.

\section{CONCLUSION}

Our understanding of the barrier function of the intestinal mucosa and its changes in IBD patients has improved significantly in recent years. In this spirit, the production of cytokines, enzymes, and other molecules by the single gut strengthens the mucous membrane and makes it an expanding field of research. Probiotic technology, such as LAB, is an excellent tool for molecules directly on the mucosa without causing side effects or systemic disadvantages of substances. They are harmless to humans and have been closely associated with humans for centuries. In addition to colonizing those used without a permanent intestine and designed so as not to suffer damage Wednesday, it is strictly controlled by the introduction of the desired molecule. For this reason, they have clinical applications of LAB for the supply of extended molecules. Some products, such as $L$. lactis mouthwash from manufacturer are currently in phase $2 / 3$ clinical trials. I hope others will follow soon. With the advent of deep coherence, we have also gained a new understanding of the complexity of the microbiota that colonizes our colon and its modifications in various pathologies, including IBD and infections. We currently understand that inflammation forms microbial communities in the intestines and only the strongest survive in an inflamed environment. Intestinal pathogens, such as S. Typhimurium, have acquired several growth mechanisms in inflamed intestines and compete with the microbiota. If we understand how pathogens survive in the inflamed digestive tract, we can isolate or produce probiotic strains with similar colonization properties. inflamed intestines and competition with pathogens around the niche, which shares many of the fitness factors associated with intestinal pathogens, is a natural example of such probiotics. In addition, the presence of persistent Gram-negative bacteria, in the inflamed intestinal mucosa makes these organisms attractive candidates for the delivery of molecules when colonization required in a long-term environment is extremely hostile.

The authors declare no conflicts of interest.

\section{CONFLICT OF INTEREST}

\section{ACKNOWLEDGMENT}

The author support from the department of biological sciences, faculty of Science, King Abdulaziz University, Jeddah, Saudi Arabia.

\section{REFERENCES}

[1] FAO/WHO. 2001. Health and nutritional properties of probiotics in food including powder milk with live lactic acid bacteria. Cordoba, Argentina, October 1-4. 
[2] Anukam KC, Reid G. 2008. Probiotics: 100 years (1907- 2007) after Elie Metchnikoff 's observations. In Communicating current research and educational topics and trends in applied microbiology, 2007 ed. (ed. Mendez-vilas A), pp. 466-474. Formatex.org, Spain.

[3] Marco ML, Pavan S, Kleerebezem M. 2006. Towards understanding molecular modes of probiotic action. Curr Opin Biotechnol 17: 204-210.

[4] Nissle A. 1959. Explanations of the significance of colonic dysbacteria \& the mechanism of action of E. coli therapy (mutaflor). Medizinische 4: 1017-1022.

[5] Mollenbrink M, Bruckschen E. 1994. Treatment of chronic constipation with physiologic Escherichia coli bacteria. Results of a clinical study of the effectiveness and tolerance of microbiological therapy with the E. coli Nissle 1917 strain (Mutaflor). Med Klin (Munich) 89: 587-593.

[6] Floch MH, Walker WA, Madsen K, Sanders ME, Macfarlane GT, Flint HJ, Dieleman LA, Ringel Y, Guandalini S, Kelly CP, et al. 2011. Recommendations for probiotic use-2011 update. J Clin Gastroenterol 45 (Suppl): S168-S171.

[7] Cotter PD, Hill C, Ross RP. 2005. Bacteriocins: Developing innate immunity for food. Nat Rev Microbiol 3: $777-788$.

[8] Servin AL. 2004. Antagonistic activities of lactobacilli and bifidobacteria against microbial pathogens. FEMS Microbiol Rev 28: $405-440$.

[9] Judith Behnsen, Elisa Deriu, Martina Sassone-Corsi, and Manuela Raffatellu Probiotics: Properties, Examples, and Specific Applications, Cold Spring Harb Perspect Med 2013;3: a010074.

[10] Nikhil Kumar, Francesco Marotta, Tejpal Dhewa, Vijendra Mishra, Vikas Kumar and Aarti Bharadwaj MANAGEMENT OF ORAL HEALTH THROUGH NOVEL PROBIOTICS: A REVIEW, International Journal of Probiotics and Prebiotics Vol. 12, No. 3, pp. 109-114, 2017

[11] Hooper LV, Gordon JI. 2001. Commensal host-bacterial relationships in the gut. Science 292: 1115-1118.

[12] DiBaise JK, Zhang H, Crowell MD, Krajmalnik-Brown R, Decker GA, Rittmann BE. 2008. Gut microbiota and its possible relationship with obesity. Mayo Clin Proc 83: 460-469.

[13] Spor A, Koren O, Ley R. 2011. Unravelling the effects of the environment and host genotype on the gut microbiome. Nat Rev Microbiol 9: 279-290.

[14] Arumugam M, Raes J, Pelletier E, Le Paslier D, Yamada T, Mende DR, Fernandes GR, Tap J, Bruls T, Batto JM, et al. 2011. Enterotypes of the human gut microbiome. Nature 473: 174-180.

[15] Wu GD, Chen J, Hoffmann C, Bittinger K, Chen YY, Keilbaugh SA, Bewtra M, Knights D, Walters WA, Knight R, et al. 2011. Linking long-term dietary patterns with gut microbial enterotypes. Science 334: 105-108.

[16] Mahowald MA, Rey FE, Seedorf H, Turnbaugh PJ, Fulton RS, Wollam A, Shah N, Wang C, Magrini V, Wilson RK, et al. 2009. Characterizing a model human gut microbiota composed of members of its two dominant bacterial phyla. Proc Natl Acad Sci 106: 5859-5864.

[17] Vinolo MA, Rodrigues HG, Nachbar RT, Curi R. 2011. Regulation of inflammation by short chain fatty acids. Nutrients 3: 858-876.

[18]FAO/WHO (2002) Guidelines for the evaluation of probiotics in foods. Food and Agriculture Organization of the United Nations and World Health Organization Expert Consultation Report. Food and Agricultural Organization of the United Nations and World Health Organization Working Group Report (online).

[19] Fuller R (1989) Probiotics in man and animals. J Appl Bacteriol 66: 65-378.

[20] Salminen SJ, Gueimonde M \& Isolauri E (2005) Probiotics that modify disease risk. J Nutr 135: 1294-1298.

[21] Nagpal R, Behare PV, Kumar M et al. (2011) Milk, milk products and disease-free health: an updated overview. Crit Rev Food Sci Nutr 99999: 1549-7852. (doi: 10.1080/ 10408398.2010.500231) [Epub ahead of print].

[22] Parvez S, Malik KA, Ah Kang S \& Kim HY (2006) Probiotics and their fermented food products are beneficial for health. J Appl Microbiol 100: 1171-1185.

[23] Tsukahara T, Tsukahara R, Yasuda S et al. (2006) Different residues mediate recognition of 1-O-oleyllysophosphatidic acid and rosiglitazone in the ligand binding domain of peroxisome proliferator-activated receptor gamma. $\mathrm{J}$ Biol Chem 281: 3398-3407.

[24]Forestier C, De Champs C, Vatoux C \& Joly B (2001) Probiotic activities of Lactobacillus casei rhamnosus: in-vitro adherence to intestinal cells and antimicrobial properties. Res Microbiol 152: 167-173.

[25] Ohashi Y \& Ushida K (2009) Health-beneficial effects of probiotics: its mode of action. Anim Sci J 80: $361-371$.

[26] Collins JK, Thornton G \& Sulliva GO (1998) Selection of probiotic strains for human application. Int Dairy J 8: 487-490.

[27] Desjardins ML \& Roy D (1990) Growth of Bifidobacteria and their enzyme profiles. J Dairy Sci 73: $299-307$.

[28] Dave R \& Shah NP (1997) Viability of probiotic bacteria in yoghurt made from commercial starter cultures. Int Dairy J 7: $31-41$.

[29] Goldin BR \& Gorbach SL (1980) Effect of Lactobacillus acidophilus dietary supplements on 1,2 dimethylhydrazine dihydrochloride-induced intestinal cancer in rats. J Natl Cancer Inst 64: 263-265.

[30] Goldin BR \& Gorbach SL (1984) The effect of milk and lactobacillus feeding on human intestinal bacterial enzyme activity. Am J Clin Nutr 39: 756-761.

[31] Kumar M, Verma V, Nagpal R, Kumar A, Behare PV, Singh B \& Aggarwal PK (2011a) Anticarcinogenic effect of probiotic fermented milk and Chlorophyllin on aflatoxin-B1 induced liver carcinogenesis in rats. Br J Nutr 107: 1006-1016.

[32] Kumar M, Verma V, Nagpal R, Kumar A, Gautam SK, Behare PV, Grover CR \& Aggarwal PK (2011b) Effect of probiotic fermented milk and chlorophyllin on gene expressions and genotoxicity during AFB1-induced hepatocellular carcinoma. Gene 490: 54-59. 
[33] Pedrosa MC, Golner BB, Goldin BR, Barakat S, Dallal GE \& Russell RM (1995) Survival of yogurt-containing organisms and Lactobacillus gasseri (ADH) and their effect on bacterial enzyme activity in the gastrointestinal tract of healthy and hypochlorhydric elderly subjects. Am J Clin Nutr 61: 353-359.

[34] Lidbeck A, Geltner AU, Orrhage KM, Ottova L, Brismar B, Gustafsson JA, Rafter JJ \& Nord CE (1991) Impact of Lactobacillus acidophilus supplements on the fecal microflora and soluble fecal bile acids in colon cancer patients. Microb Ecol Health Dis 4: 81-88.

[35] Drisko JA, Giles CK \& Bischoff BJ (2003) Probiotics in health maintenance and disease prevention. Altern Med Rev 8: 143155.

[36] Hirayama K \& Rafter J (2000) The role of probiotic bacteria in cancer prevention. Microbes Infect 2: $681-686$.

[37] Li W \& Li CB (2003) Lack of inhibitory effects of lactic acid bacteria on 1,2-dimethylhydrazine-induced colon tumors in rats. World J Gastroenterol 9: 2469-2473.

[38] Ravinder Nagpal, Ashwani Kumar, Manoj Kumar, Pradip V. Behare4, Shalini Jain \& Hariom Yadav Probiotics, their health benefits and applications for developing healthier foods: a review, FEMS Microbiol Lett 334 (2012) 1-15.

[39] Paula J.P. Espitia Rejane A. Batista, Henriette M.C. Azeredo, Caio G. Otoni Probiotics and their potential applications in active edible films and coatings, Food Research International V. 90, 2016, Pages 42-52

[40] Hansen CH, Nielsen DS, Kverka M, Zakostelska Z, Klimesova K, Hudcovic T, Tlaskalova-Hogenova H, Hansen AK (2012) Patterns of early gut colonization shape future immune responses of the host. PLoS One 7:e34043.

[41] Biasucci G, Rubini M, Riboni S, Morelli L, Bessi E, Retetangos C (2010) Mode of delivery affects the bacterial community in the newborn gut. Early Hum Dev 86:13-15.

[42] Penders J, Thijs C, van den Brandt PA, Kummeling I, Snijders B, Stelma F, Adams H, van Ree R, Stobberingh EE (2006) Gut microbiota composition and development of atopic manifestations in infancy: the KOALA Birth Cohort Study. Gut 56:661-667.

[43] Tham CSC, Peh KK, Bhat R, Liong MT (2011) Probiotic properties of bifidobacteria and lactobacilli isolated from local dairy products. Ann Microbiol 62:1079-1087.

[44] Coppa G, Gabrielli O (2008) Human milk oligosaccharides as prebiotics. In: Versalovic J, Wilson M (eds) Therapeutic microbiology: probiotics and related strategies. American Society for Microbiology Press, Washington, pp 131-146.

[45] Solís G, de Los Reyes-Gavilan CG, Fernández N, Margolles A, Gueimonde M (2010) Establishment and development of lactic acid bacteria and bifidobacteria microbiota in breast milk and the infant gut. Anaerobe 16:307-310.

[46] Arboleya S, Ruas-Madiedo P, Margolles A, Solís G, Salminen S, de Los Reyes-Gavilán CG, Gueimonde M (2011) Characterization and in vitro properties of potentially probiotic Bifidobacterium strains isolated from breast-milk. Int J Food Microbiol 149:28-36.

[47] Barrett E, Guinane C, Ryan A, Dempsey E, Murphy B, O’Toole P, Fizgerarld G, Cotter P, Ross P, Stanton C (2013) Microbiota diversity and stability of the preterm neonatal ileum and colon of two infants. MicrobiolOpen 2:215-225.

[48] Diana Di Gioia \& Irene Aloisio \& Giuseppe Mazzola \& Bruno Biavati Bifidobacteria: their impact on gut microbiota composition and their applications as probiotics in infants, Appl Microbiol Biotechnol (2014) 98:563-577.

[49] Scherlach, K., Graupner, K., and Hertweck, C. (2013). Molecular bacteria-fungi interactions: effects on environment, food, and medicine. Annu. Rev. Microbiol. 67, 375-397. doi: 10.1146/annurev-micro-092412-155702.

[50] Dalié, D. K. D., Deschamps, A. M., and Richard-Forget, F. (2010). Lactic acid bacteria - Potential for control of mould growth and mycotoxins: a review. Food Control 21, 370-380. doi: 10.1016/j.foodcont.2009.07.011.

[51] Fleet, G. (2003). Yeast interactions and wine flavour. Int. J. Food Microbiol. 86, 11-22. doi: 10.1016/S0168-1605(03)002459.

[52] Rouse, S., Harnett, D., Vaughan, A., and van Sinderen, D., (2008). Lactic acid bacteria with potential to eliminate fungal spoilage in foods. J. Appl. Microbiol. 104, 915-923. doi: 10.1111/j.1365-2672.2007.03619.x.

[53] Viljoen, B. C. (2001). The interaction between yeasts and bacteria in dairy environments. Int. J. Food Microbiol. 69, 37-44. doi: 10.1016/S0168-1605(01)00570-0.

[54] Lopitz-Otsoa, F., Ramenteria, A., Elguezabal, N., and Garaizar, J. (2006). Kefir: a symbiotic yeast-bacteria community with alleged healthy communities. Rev. Iberoam. Micol. 23, 67-74. doi: 10.1016/S1130-1406(06)70016-X.

[55] Valerio, F., Favilla, M., De Bellis, P., Sisto, A., de Candia, S., and Lavermicocca, P. (2009). Antifungal activity of strains of lactic acid bacteria isolated from a semolina ecosystem against Penicillium roqueforti, Aspergillus niger and Endomyces fibuliger contaminating bakery products. Syst. Appl. Microbiol. 32, 438-448. doi: 10.1016/j.syapm.2009.01.004.

[56] Viljoen, B. C. (2001). The interaction between yeasts and bacteria in dairy environments. Int. J. Food Microbiol. 69, 37-44. doi: 10.1016/S0168-1605(01)00570-0

[57] Ryan, L. A. M., Zannini, E., Dal Bello, F., Pawlowska, A., Koehler, P., and Arendt, E. K. (2011). Lactobacillus amylovorus DSM 19280 as a novel food-grade antifungal agent for bakery products. Int. J. Food Microbiol. 146, $276-283$. doi: 10.1016/j.ijfoodmicro.2011.02.036.

[58] Dalié, D. K. D., Deschamps, A. M., and Richard-Forget, F. (2010). Lactic acid bacteria - Potential for control of mould growth and mycotoxins: a review. Food Control 21, 370-380. doi: 10.1016/j.foodcont.2009.07.011.

[59] Goerges, S., Aigner, U., Silakowski, B., and Scherer, S. (2006). Inhibition of Listeria monocytogenes by food-borne yeasts. Appl. Environ. Microbiol. 72, 313-318. doi: 10.1128/AEM.72.1.313-318.2006.

[60] Jard, G., Liboz, T., Mathieu, F., Guyonvarc'h, A., and Lebrihi, A. (2011). Review of mycotoxin reduction in food and feed: from prevention in the field to detoxification by adsorption or transformation. Food Addit. Contam. A 28, 1590-1609. doi: 10.1080/19440049.2011.595377. 
[61] Ibrahim, A. S., Gebremariam, T., Liu, M., Chamilos, G., Kontoyiannis, D., Mink, R., et al. (2008). Bacterial endosymbiosis is widely present among zygomycetes but does not contribute to the pathogenesis of mucormycosis. J. Infect. Dis. 198, 1083-1090. doi: 10.1086/591461.

[62] Scherlach, K., Graupner, K., and Hertweck, C. (2013). Molecular bacteria-fungi interactions: effects on environment, food, and medicine. Annu. Rev. Microbiol. 67, 375-397. doi: 10.1146/annurev-micro-092412-155702.

[63] Jard, G., Liboz, T., Mathieu, F., Guyonvarc'h, A., and Lebrihi, A. (2011). Review of mycotoxin reduction in food and feed: from prevention in the field to detoxification by adsorption or transformation. Food Addit. Contam. A 28, 1590-1609. doi: 10.1080/19440049.2011.595377.

[64]Lackner, G., and Hertweck, C. (2011). Impact of endofungal bacteria on infection biology, food safety, and drug development. PLoS Pathog. 7, 5-8. doi: 10.1371/journal.ppat.1002096.

[65] Buckle, K. A., and Kartadarma, E. (1990). Inhibition of bongkrek acid and toxoflavin production in tempe bongkrek containing Pseudomonas cocovenenans. J. Appl. Bacteriol. 68, 571-576. doi: 10.1111/j.1365-2672.1990.tb05222.x.

[66] Tiroyaone Shimane Tshikantwa, Muhammad Wajid Ullah, Feng He and Guang Yang Current Trends and Potential Applications of Microbial Interactions for Human Welfare, Frontiers in Microbiology, 2018 | V. 9 | Article 11565 Issue: 07. 